\title{
Type B Insulin Resistance Presenting as Hypoglycemia
}

\author{
Charles Meade, Steve Stanek, Ho-man Yeung, Isabel Marcano, David Baumgarten, Hal Kominsky, Xiaoying \\ Deng*
}

Department of Medicine, Lewis Katz School of Medicine, Temple University, USA

Submission: January 24, 2018; Published: February 28, 2018

*Corresponding author: Xiaoying Deng, Department of Medicine, Lewis Katz School of Medicine, Temple University, USA,

Email: xiaoying.deng@tuhs.temple.edu

\section{Case Report}

A 60-year-old African-American woman with a past medical history significant for systemic lupus erythematosus and thyroglobulin peroxidase-positive Hashimoto's thyroiditis presented in September 2015 with nausea, coffee ground emesis, shortness of breath, and chest pain, blood glucose less than $10 \mathrm{mg} / \mathrm{ml}$. She had no glucose disorder prior to June 2013, and she weighed 259 pounds with BMI of 44.5 at that time. By September 2013, significant weight loss caused her BMI to become 31.7. DM was suspected and HbA1C was 6.3\%. By August 2014, hemoglobin A1c was $14.4 \%$ and BMI 26.9. One month later, she was admitted for symptomatic hypoglycemia. Her workup revealed hyperinsulinemia $280 \mathrm{uIU} / \mathrm{mL}$ with normal C-peptide levels; repeated plasma insulin 133uIU/ $\mathrm{mL}, \mathrm{C}$-peptide levels $0.2 \mathrm{ng} / \mathrm{mL}$, B-hydroxybutyrate elevated to $7.7 \mathrm{mg} / \mathrm{dL}$, and corresponding hypoglycemia $50 \mathrm{mg} / \mathrm{dL}$. Her insulin autoantibodies and image studies were negative. She was treated with steroids and IVIG 2 grams $/ \mathrm{kg}$ per day for 5 days. Her hypoglycemia resolved, then she developed persistent hyperglycemia, requiring a regimen of $14 \mathrm{U}$ long-acting insulin with $4 \mathrm{U}$ short-acting insulin with meals.

At this admission, she was placed on continuous D10, then D5 intravenous infusion. She continued to be hypoglycemic at $30 \mathrm{~s}-40 \mathrm{smg} / \mathrm{dL}$, occasionally dropping to $12-13 \mathrm{mg} / \mathrm{dL}$. Insulin measurement on hospital Day 3 showed $1747 \mathrm{uIU} / \mathrm{ml}$, C-peptide $25.0 \mathrm{ng} / \mathrm{mL}$ with blood glucose $<10 \mathrm{mg} / \mathrm{dL}$. On hospital day 4 , her creatinine increased to $5.89 \mathrm{mg} / \mathrm{dl}$, lupus caused nephritis was suspected, and Solumedrol 500mg IV daily were given for 3 days. Her kidney biopsy suggested acute tubular necrosis, not lupus nephritis. She remained hypoglycemic. On Day 7, another 3 days of $1000 \mathrm{mg}$ solumedrol intravenous daily were given, allowing her to be weaned off Dextrose. She still had difficulty maintaining glucose above $60 \mathrm{mg} / \mathrm{dl}$. A diagnosis of Type B insulin resistance was made. Octreotide, $50 \mathrm{mcg}$ every 8 hours for 4 days, was administered; she continued to experience hypoglycemic episodes at $30-40 \mathrm{mg} / \mathrm{dL}$. Octreotide was discontinued, and a trial of intravenous immunoglobulin began at $1 \mathrm{mg} / \mathrm{kg}$, $40 \mathrm{~g} / \mathrm{day}$, for 3 days. Following IVIG treatment, her blood glucose levels finally stabilized between $90-110 \mathrm{mg} / \mathrm{dL}$, and she was discharged home.

First described by Kahn et al. in 1976 [1], Type B insulin resistance is a rare disorder of glucose homeostasis distinguished by the presence of autoantibodies directed against the insulin receptor. The original case series describes 3 female patients presenting with marked hyperglycemia and insulin resistance with autoantibodies. All had acanthosis nigricans, weight loss, spontaneous remission of glucose intolerance, and association with other autoimmune disorders.

Type B insulin resistance has since been further characterized. Arioglu et al. compiled 24 cases [2]. 88\% of cases were African-American, 21 presented with hyperglycemia, and strong associations with concomitant autoimmune disorder and particularly SLE were noted. Patients presenting with hyperglycemia also had weight loss and aconthosis nigricans. Titers of anti-insulin receptor autoantibodies disappeared spontaneously in about $1 / 3$ cases. Three patients presented with initial hypoglycemia. Another three patients experienced spontaneous hypoglycemia after prolonged courses of hyperglycemia (3-21 months). Conversion from hyperglycemia to hypoglycemia was identified as a poor prognostic factor in the presence of Type B insulin resistance. All three of those patients died from hypoglycemia-related complications.

While the mechanism for refractory hyperglycemia in Type B insulin resistance is understood as autoantibody antagonist activity at the insulin receptor, the mechanism for the conversion from hyperglycemia to hypoglycemia is less clear. Several hypotheses have been put forth. Animal studies have shown a concentration-dependent antagonist or agonist activity; given the limited number of cases, evidence is circumstantial $[3,4]$. Other proposed mechanisms include a change in expression levels and affinity of the insulin receptor secondary to autoantibody exposure, and a possible evolution in the type of antibody expressed from an early to a late antagonist type $[3,4]$. 
Recently, Xhang et al. compiled 67 cases of Type B insulin resistance and examined treatment strategies [5]. For hyperglycemic patients, liberal use of insulin was recommended to control blood glucose. In 39 cases, a relatively large insulin doses were required to control hyperglycemia. Some patients required immunosuppression directed against insulin receptor antibodies. In patients with poor initial response, glucocorticoids were typically followed by more aggressive immunosuppressive therapies. Recommended as standardized treatment, Rituximab, cyclophosphamide, and pulse corticosteroids were shown to reduce autoantibody titers in 7 cases. In patients with very high titers, plasmapheresis was shown to have rapid effect and poor long-term efficacy, thus potentially useful in combination therapy. Having observed its effectiveness in 2 cases, the authors suggested IVIG as a reasonable first-line therapy.

Our patient, an adult African-American female with SLE and Hashimoto's thyroiditis, illustrates common features present in Type B insulin resistance as well as the difficulty of treating under-characterized hyperglycemic to hypoglycemic conversion. Most cases of Type B insulin resistance in the literature fit a similar demographic profile and a history of autoimmune disorders. Pulse corticosteroids and IVIG eventually succeeded in resolving hypoglycemia and restoring glycemic control. Other potentially successful therapies include immunosuppressive therapy with rituximab and cyclophosphamide, but IVIG and high-dose corticosteroids remain optimal first line agents. Given the risk for further hypoglycemic episodes, we hope that discharge with implantable continuous glucose monitoring will mitigate the mortality observed in some cases. Our case reaffirms the importance of considering a diagnosis of autoimmune insulin syndromes in a differential for workup of either hyper- or hypoglycemia, especially given a background of other autoimmune diseases. With regards to underlying pathophysiology, treatment strategies for Type B insulin resistance must continue to be refined.

\section{References}

1. Kahn CR, Flier JS, Bar RS, Archer JA, Gorden P, et al. (1976) The syndromes of insulin resistance and acanthosis nigricans. Insulinreceptor disorders in man. N Engl J Med 294(14): 739- 745.

2. Arioglu E, Andewelt A, Diabo C, Bell M, Taylor SI, et al. (2002) Clinical course of the syndrome of autoantibodies to the insulin receptor (type B insulin resistance): a 28-year perspective. Medicine (Baltimore) 81(2): 87-100.

3. Lupsa BC, Chong AY, Cochran EK, Soos MA, Semple RK, et al. (2009) Autoimmune forms of hypoglycemia. Medicine (Baltimore) 88(3): 141153.

4. Redmon JB, Nuttall FQ (1999) Autoimmune hypoglycemia. Endocrinol Metab Clin North Am 28(3): 603-618.

5. Zhang S, Wang G, Wang J (2013) Type B insulin resistance syndrome induced by systemic lupus erythematosus and successfully treated with intravenous immunoglobulin: case report and systematic review. Clin Rhematol 32(2): 181-188.

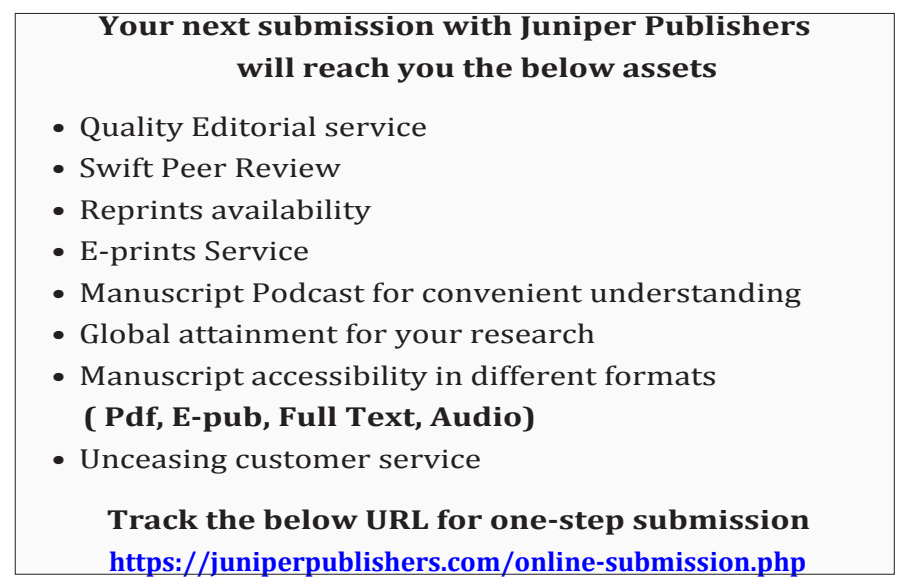

